\title{
Evaluating Question Answering Evaluation
}

\author{
Anthony Chen ${ }^{1}$, Gabriel Stanovsky ${ }^{2}$, Sameer Singh $^{1}$, and Matt Gardner ${ }^{3}$ \\ ${ }^{1}$ University of California, Irvine, USA \\ ${ }^{2}$ Allen Institute for Artificial Intelligence, Seattle, Washington, USA \\ ${ }^{3}$ Allen Institute for Artificial Intelligence, Irvine, California, USA \\ anthony . chen@uci.edu
}

\begin{abstract}
As the complexity of question answering (QA) datasets evolve, moving away from restricted formats like span extraction and multiplechoice (MC) to free-form answer generation, it is imperative to understand how well current metrics perform in evaluating QA. This is especially important as existing metrics (BLEU, ROUGE, METEOR, and F1) are computed using $n$-gram similarity and have a number of well-known drawbacks. In this work, we study the suitability of existing metrics in QA. For generative QA, we show that while current metrics do well on existing datasets, converting multiple-choice datasets into free-response datasets is challenging for current metrics. We also look at span-based QA, where $\mathrm{F}_{1}$ is a reasonable metric. We show that $F_{1}$ may not be suitable for all extractive QA tasks depending on the answer types. Our study suggests that while current metrics may be suitable for existing QA datasets, they limit the complexity of QA datasets that can be created. This is especially true in the context of free-form QA, where we would like our models to be able to generate more complex and abstractive answers, thus necessitating new metrics that go beyond $n$-gram based matching. As a step towards a better QA metric, we explore using BERTScore, a recently proposed metric for evaluating translation, for QA. We find that although it fails to provide stronger correlation with human judgements, future work focused on tailoring a BERT-based metric to QA evaluation may prove fruitful.
\end{abstract}

\section{Introduction}

Question answering (QA) has emerged as a burgeoning research field driven by the availability of large datasets. These datasets are built to test a variety of reading comprehension skills such as multihop (Welbl et al., 2017), numerical (Dua et al.,
Context: ... After Peter returns, they eventually figure out her proper care, right down to diaper changes, baths, and feedings. The next day, two men (who are drug dealers) arrive at the apartment to pick up the package...

Question: Who comes to pick up the package the next day?

Gold Answers: drug dealers, the drug dealer Prediction: two men

Human Judgement: 5 out of 5

ROUGE-L: 0

METEOR: 0

(a) Example from the generative NarrativeQA dataset.

Context: ... David got five exercise tips from his personal trainer, tip A, tip B ... Tip A involves weight lifting, but tip B does not involve weight lifting ...

Question: In which tip the skeletal muscle would not be bigger, tip A or tip B?

Gold Answers: tip B

Prediction: tip A

Human Judgement: 1 out of 5

F1: 0.66

(b) Example from the span-based ROPES dataset.

Figure 1: Examples where existing $\boldsymbol{n}$-gram based metrics fail to align with human judgements. Human judgements are between 1 and 5. (a) illustrates that because existing metrics do not use the context, they fail to capture coreferences. (b) illustrates that changing a single token can make a prediction incorrect while $F_{1}$ assigns a non-zero score.

2019), and commonsense (Talmor et al., 2018) reasoning. A key component of a QA dataset is the evaluation metric associated with it, which aims to automatically approximate human accuracy judgments of a predicted answer against a gold answer.

The metrics used to evaluate QA datasets have a number of ramifications. The first is that they drive research focus. Models that rank higher on a leaderboard according to a metric will receive 
more community attention. The second is that just as good datasets drive model development, good metrics drive dataset development. As QA datasets become more complex and models are expected to produce more free-form and abstract answers, it is crucial that the metrics we use are able to assign scores that accurately reflect human judgements. Despite the value of metrics as drivers of research, a comprehensive study of QA metrics across a number of datasets has yet to be completed. This is important as present metrics are based on $n$-gram matching, which have a number of shortcomings (Figure 1).

In this work, we survey the landscape of evaluation metrics for QA and study how well current metrics approximate (i.e. correlate with) human judgements. We conduct our study on three datasets: NarrativeQA (Kociský et al., 2017), ROPES (Lin et al., 2019), and SemEval-2018 Task 11 (Ostermann et al., 2018). For the generative NarrativeQA dataset, we find that existing metrics provide reasonable correlation with human accuracy judgements while still leaving considerable room for improvement. We also study the span-based ROPES dataset, finding that it presents an interesting case where F1 struggles due to the high overlap in right and wrong answers. Finally, we convert the multiple-choice SemEval-2018 Task 11 dataset into a generative QA dataset. This produces a more difficult generative QA dataset compared to NarrativeQA as answers in SemEval are often more free-form in nature and have less overlap with the context. Here we find existing $n$-gram based metrics perform considerably worse in comparison to NarrativeQA.

These results signify that as QA systems are expected to perform more free-form answer generation, new metrics will be required. In exploring other metrics that go beyond $n$-gram matching, we study the recently proposed BERTScore. BERTScore computes a score by leveraging contextualized word representations, allowing it to go beyond exact match and capture paraphrases better. We find that it falls behind existing metrics on all three datasets. We propose a potential step in constructing a better QA metric by extending BERTScore to incorporate the context and the question when computing the similarity between two answers. We show that extending BERTScore in this way slightly improves results when evaluating generative QA, though not to an extant that is sta- tistically significant. Overall, our results indicate that studying the evaluation of QA is an underresearched area with substantial room for further experimentation.

\section{Metrics}

We provide a summary of popular $n$-gram based metrics, as well as sentence mover's similarity, BERTScore, and an extension of BERTScore which we call conditional BERTScore. In this work, we study all mentioned metrics in the context of question answering.

BLEU is a precision-based metric developed for evaluating machine translation (Papineni et al., 2001). BLEU scores a candidate by computing the number of $n$-grams in the candidate that also appear in a reference. $n$ is varied from 1 up to a specified $N$ and the scores for varying $n$ are aggregated with a geometric mean. In this work, we look at BLEU-1 and BLEU-4, where $N=1$ and $N=4$ respectively.

METEOR is an F-measure metric developed for evaluating machine translation which operates on unigrams (i.e. tokens) (Banerjee and Lavie, 2005). METEOR first creates an alignment by attempting to map each token in a candidate to a token in a reference (and vice versa). A token is aligned to another token if they are the same, are synonyms, or their stems match. The alignment is aggregated into precision and recall values, which are combined into an F-measure score in which more weight is given to recall.

ROUGE is an F-measure metric designed for evaluating translation and summarization (Lin, 2004). There are a number of variants of ROUGE however in this work we focus on ROUGE-L. ROUGE-L is computed based on the longest common subsequence (LCS), which searches for the longest co-occurring set of tokens common to both reference and candidate. An advantage of ROUGE$\mathrm{L}$ is that no predefined $n$-gram size is required.

$\mathbf{F}_{1}$ While the previously mentioned metrics have been adapted for evaluating generative question answering, $F_{1}$ has been generally reserved for evaluating span-based question answering (Rajpurkar et al., 2016). It is computed over tokens in the candidate and reference.

Sentence Mover's Similarity (SMS) is a recent metric based on earth mover's distance for evaluated multi-sentence texts such as machinegenerated summaries (Clark et al., 2019) . ${ }^{1}$ SMS

\footnotetext{
${ }^{1}$ https://github.com/eaclark07/sms
} 
first computes an embedding for each sentence in a document as an average its ELMo word representations (Peters et al., 2018). A linear program is then solved to obtain the distance of "moving" a candidate document's sentences to match a reference document. SMS has shown better results over ROUGE-L in evaluating generated summaries and student essays.

BERTScore is recent metric for evaluating translation (Zhang et al., 2019). ${ }^{2}$ BERTScore first obtains BERT representations of each word in the candidate and reference by feeding the candidate and reference through a BERT model separately. An alignment is then computed between candidate and reference words by computing pairwise cosine similarity. This alignment is then aggregated in to precision and recall scores before being aggregated into a (modified) F1 score that is weighted using inverse-document-frequency values. BERTScore has been shown to align better to human judgements in evaluating translation compared to existing metrics. Additionally, because it uses word representations and not exact match, BERTScore has also been shown to capture paraphrases better than existing metrics. We include BERTScore and SMS in this work because they have not yet been studied in the context of QA.

Conditional BERTScore A key difference between machine translation and QA is that determining the correctness of a predicted answer requires using information from the context and question (Figure 1a). While BERTScore can potentially handle phenomena like paraphrases better than existing metrics, it still overlooks the context and question. We propose an extension to BERTScore that incorporates the context and question when calculating the answer word representations. More specifically, we concatenate the context, question, and answer delineated by BERT separator tokens as the input to BERT. We then extract the BERT representations of the answer words and compute BERTScore. In this way, the representation of the answer words are conditioned (i.e. contextualized) with the context and question.

\section{Datasets}

We describe the three QA datasets we use with examples in Table 1.

NarrativeQA is a generative QA dataset on books and movie scripts (Kociský et al., 2017). The

\footnotetext{
${ }^{2}$ https://github.com/Tiiiger/bert_score
}

contexts are plot summaries taken from Wikipedia and each question has two reference answers. The official evaluation metrics of NarrativeQA are BLEU-1, BLEU-4, METEOR, and ROUGE-L.

SemEval-2018 Task 11 (which we refer to as SemEval for brevity) is a multiple-choice QA dataset which focuses on commonsense reasoning about everyday scenarios (Ostermann et al., 2018). We convert this into a generative QA dataset by using the correct answer choice as a target for a generative QA system. We hypothesize that this results in a more difficult generative QA dataset compared to NarrativeQA as a number of the answers in the SemEval dataset have no overlap with the question or context.

ROPES is a recent span-based QA dataset with questions that focus on cause-and-effect relationships (Lin et al., 2019). Each question is accompanied by a background passage with auxiliary information and a situation passage. We concatenate the background and situation to use as the context. The official evaluation metric of ROPES is $F_{1}$. A unique characteristic of ROPES is that questions generally present two possible answer choices, one of which is incorrect (Table 1). Because incorrect and correct answers often have some $n$-gram overlap, we believe $F_{1}$ will struggle to accurately assign scores (Figure 1b).

\section{Models}

We describe the models used to generate predictions for our datasets. These models have publicly available code and have reasonable performance compared to the current state-of-the-art models.

Multi-hop Point Generator For NarrativeQA and SemEval, we use a multi-hop pointer generator (MHPG) model (Bauer et al., 2018) ${ }^{3}$. MHPG represents its input using ELMo embeddings. The embeddings are then fed into a sequence of BiDAF (Seo et al., 2017) cells, where the output of one BiDAF cell is fed as the input into another BiDAF cell. This allows multi-hop reasoning over the context. The output layer consists of a generative decoder with a copying mechanism. We evaluate MHPG's predictions using BLEU-1, BLEU-4, ROUGE-L, METEOR, SMS, BERTScore and Conditional BERTScore.

BERT For ROPES, we finetune BERT as a span based QA model following the procedure used for

\footnotetext{
${ }^{3}$ https: //github. com/yicheng-w/ CommonSenseMultiHopQA
} 


\begin{tabular}{|c|c|c|c|c|}
\hline Dataset & \# QA Pairs & Context & Question & Gold Answer \\
\hline NarrativeQA & 32,747 & $\begin{array}{l}\text {... An earthquake triggers the transfer, bring- } \\
\text { ing the ship into the present ... After carrying } \\
\text { the men through hyperspace, the ship lands on } \\
\text { a planet where faltering robots refuel the ship } \\
\text {.. }\end{array}$ & $\begin{array}{l}\text { How were the men } \\
\text { able to find fuel for } \\
\text { the spaceship? }\end{array}$ & $\begin{array}{l}\text { The first planet had } \\
\text { robots that fueled } \\
\text { the ship. }\end{array}$ \\
\hline SemEval & 9,731 & $\begin{array}{l}\text { One evening, I noticed my alarm clock had } \\
\text { stopped working ... I lifted the plastic cover } \\
\text { and checked what batteries it required: two } \\
\text { AA-sized batteries ... }\end{array}$ & $\begin{array}{l}\text { Why did they throw } \\
\text { away the old batter- } \\
\text { ies? }\end{array}$ & $\begin{array}{l}\text { They were no longer } \\
\text { useful }\end{array}$ \\
\hline ROPES & 11,202 & $\begin{array}{l}\ldots \text { A catalyst is a chemical that speeds up } \\
\text { chemical reactions ... [Mark] conducts two } \\
\text { tests, test } A \text { and test } B \text {, on an organism. In test } \\
\text { A he reduces catalysts from the organism, but } \\
\text { in test } B \text { he induces catalysts in the organism } \\
\ldots\end{array}$ & $\begin{array}{l}\text { Which test would } \\
\text { see reactions taking } \\
\text { place slower, test A } \\
\text { or test B? }\end{array}$ & test $\mathrm{A}$ \\
\hline
\end{tabular}

Table 1: Examples for the datasets we use in our study. The \# of QA Pairs column refers to the number of QA pairs in the training sets.

SQuAD (Devlin et al., 2019). We evaluate BERT's predictions using $\mathrm{F}_{1}$, SMS, BERTScore, and Conditional BERTScore.

\section{Evaluating QA Metrics using Human Judgements}

\subsection{Collecting Human Judgements}

After training our models on the three datasets, we extract 500, 500, and 300 data points from the validation sets of NarrativeQA, ROPES, and SemEval, respectively, along with the model predictions. When extracting data points to label, we filter out data points where the predicted answer exactly matches the gold answer. This filtering step is done as we are interested on how well metrics do when it cannot resort to exact string matching.

For the extracted data points, we ask annotators to rate how closely a prediction captures the same information as a gold answer. Annotations are on a scale from 1 to 5. Two of the authors annotated all data points in-house. We find strong agreement between the two annotators across the three datasets (see Table 3). We note that because we have removed exact matches, the distribution of human judgement scores is right-skewed for each dataset. This is most prominent in ROPES, where around 400 predictions are labeled as a 1.

\subsection{Correlation with Human Judgements}

We first normalize the judgements for each annotator following Blatz et al. (2004) and then average the judgements of the two annotators to obtain a single gold annotation per data point. We then com- pute the Spearman and Kendall correlation of the gold annotations to the scores assigned by automatic metrics. The correlation results are presented in Table 2.

\subsection{Discussion}

Of NarrativeQA's four evaluation metrics, METEOR aligns closest with human judgements, while leaving considerable room for improvement. ROPES proves to be a challenging dataset for F1 to evaluate. This highlights the fact that while F1 is a reasonable metric for many span-based QA datasets, the types of questions and answers can influence how well it works in practice and care should be taken when adapting evaluation metrics. For the SemEval dataset, which we converted to a generative QA dataset from a multiple-choice dataset, we find that existing metrics do considerably worse compared to NarrativeQA. This aligns with our hypothesis that more free-form generative QA datasets leads to a degradation in $n$-gram based metrics' performance. Similar to NarrativeQA, METEOR aligns best with human judgements on SemEval. We make the recommendation based on these results that for evaluating generative QA, METEOR is currently the metric that should be given the most consideration.

Both BERTScore and sentence mover's similarity fall behind the best metric for each dataset. This points to the fact that metrics that perform well for evaluating summarization and translation do not necessarily indicate success in evaluating question answering. Conditional BERTScore slightly 


\begin{tabular}{lcccccccc}
\hline \multirow{2}{*}{ Metrics } & \multicolumn{2}{c}{ NarrativeQA } & & \multicolumn{2}{c}{ SemEval } & & \multicolumn{2}{c}{ ROPES } \\
\cline { 2 - 3 } & Spearman & Kendall & & Spearman & Kendall & & Spearman & Kendall \\
\hline BLEU-1 & 0.617 & 0.483 & & 0.443 & 0.351 & & - & - \\
BLEU-4 & 0.563 & 0.433 & & 0.437 & 0.350 & & - & - \\
METEOR & $\mathbf{0 . 7 5 2}$ & $\mathbf{0 . 6 1 4}$ & & $\mathbf{0 . 6 4 2}$ & $\mathbf{0 . 5 4 2}$ & & - & - \\
ROUGE-L & 0.707 & 0.577 & & 0.570 & 0.489 & & - & - \\
Sentence Mover's Similarity & 0.474 & 0.365 & & 0.488 & 0.384 & & 0.376 & 0.307 \\
BERTScore & 0.733 & 0.573 & & 0.406 & 0.323 & & 0.448 & 0.365 \\
Conditional BERTScore & 0.741 & 0.581 & & 0.415 & 0.330 & & 0.434 & 0.353 \\
F1 & - & - & & - & - & & $\mathbf{0 . 5 9 1}$ & $\mathbf{0 . 5 4 0}$ \\
\hline
\end{tabular}

Table 2: Human Judgments and Metrics: Correlation between metrics and human judgments using Spearman's rho $(\rho)$ and Kendall's tau $(\tau)$ rank correlation coefficients. "-" indicates the metric is not used for the dataset.

\begin{tabular}{lccc}
\hline Dataset & $\kappa$ & $r$ & $\rho$ \\
\hline NarrativeQA & 0.747 & 0.951 & 0.944 \\
SemEval & 0.854 & 0.970 & 0.976 \\
ROPES & 0.962 & 0.997 & 0.992 \\
\hline
\end{tabular}

Table 3: Inter-annotator agreement computed using Cohen's kappa $(\kappa)$, Pearson correlation $(r)$, and Spearman's correlation $(\rho)$.

improves results over BERTScore on our two generative QA tasks, which is a promising sign that incorporating the context and question in a QA metric is a worthwhile pursuit. In the cases where Conditional BertScore improves over BERTScore, the gains are not statistically significant. One thing to note is that the BERT model was never exposed to context/question/answer triples during its pretraining. Finetuning a BERT model on QA datasets can potentially yield a better BERTScore-based metric.

\section{Related Work}

$\mathrm{N}$-gram based metrics such as BLEU and METEOR were originally developed and tested for evaluation of machine translation. These metrics have grown to become popular choices in evaluating all forms of natural language generation, including image captioning, question answering, and dialog systems. As these metrics continue to be used, there have been a number of papers that try to assess how suitable these metrics are for different domains. Nema and Khapra (2018) show that for question generation, $n$-gram metrics assign scores that correlate poorly to the notion of answerability (i.e., is a generated question answerable). Yang et al. (2018) study the effect of using BLEU and ROUGE in evaluating QA, focusing on yes-no and entity questions on the Chinese DuReader dataset (He et al., 2017). For these types of questions, changing a single word from a gold answer can lead to an incorrect answer. In these cases, BLEU and ROUGE assign scores that do not necessarily reflect the correctness of an answer. Our work is continuation of this line of work in assessing the quality of current metrics for use in evaluating question answering across a number of datasets.

Because of the inherent limitations of $n$-gram metrics, recent work has focused on using metrics that have been learned or are based on word representations. In image captioning, Cui et al. (2018) train a model that takes as input an image, a reference caption, and a candidate caption and learns to predict if the two captions are semantically equivalent. Using this trained model as a metric leads to better scores compared to $n$-gram based metrics. As mentioned earlier, sentence mover's similarity and BERTScore leverage contextualized word representations for evaluating summarization and translation respectively, also obtaining better results compared to existing metrics. We hope to push the evaluation of question answering in this direction and study SMS and BERTScore in the context of QA as a first step in this direction.

\section{Conclusion}

In this work, we present a systematic study of existing $n$-gram based metrics by comparing their correlation to human accuracy judgements on three QA datasets. We find that while existing metrics do fairly well on NarrativeQA, for the more free-form 
SemEval dataset, existing metrics fare significantly worse. Our results indicate that as generative QA datasets become more abstractive in nature, better metrics that go beyond $n$-gram matching will be required. We also find that $F_{1}$ struggles in evaluating the ROPES dataset, signaling that a better metric can also help improve span-based QA evaluation. In the search of a better metric, we also study BERTScore along with a conditional BERTScore that incorporates the context and question. Incorporating the context and question into BERTScore slightly improves results, indicating that a BERTbased model that uses the context and question is a promising research direction. Future work also involves the collection of more data. This includes collecting human annotations on more datasets, generating model predictions using more reading comprehension models, and also evaluating metrics on human generated answers.

\section{References}

Satanjeev Banerjee and Alon Lavie. 2005. Meteor: An automatic metric for mt evaluation with improved correlation with human judgments. In IEEvaluation@ACL.

Lisa Bauer, Yicheng Wang, and Mohit Bansal. 2018. Commonsense for generative multi-hop question answering tasks. In $A C L$.

John Blatz, Erin Fitzgerald, George F. Foster, Simona Gandrabur, Cyril Goutte, Alex Kulesza, Alberto Sanchís, and Nicola Ueffing. 2004. Confidence estimation for machine translation. In COLING.

Elizabeth Clark, Asli elikyilmaz, and Noah A. Smith. 2019. Sentence mover's similarity: Automatic evaluation for multi-sentence texts. In $A C L$.

Yin Cui, Guandao Yang, Andreas Veit, Xun Huang, and Serge J. Belongie. 2018. Learning to evaluate image captioning. 2018 IEEE/CVF Conference on Computer Vision and Pattern Recognition, pages 5804-5812.

Jacob Devlin, Ming-Wei Chang, Kenton Lee, and Kristina Toutanova. 2019. Bert: Pre-training of deep bidirectional transformers for language understanding. NAACL-HLT.

Dheeru Dua, Yizhong Wang, Pradeep Dasigi, Gabriel Stanovsky, Sameer Singh, and Matt Gardner. 2019. Drop: A reading comprehension benchmark requiring discrete reasoning over paragraphs. In $N A A C L$ HLT.

Wei He, Kai Liu, Jing Liu, Yajuan Lyu, Shiqi Zhao, Xinyan Xiao, Yuan Liu, Yizhong Wang, Hua Wu, Qiaoqiao She, Xuan Liu, Tian Wu, and Haifeng
Wang. 2017. Dureader: a chinese machine reading comprehension dataset from real-world applications. In $Q A @ A C L$.

Tomás Kociský, Jonathan Schwarz, Phil Blunsom, Chris Dyer, Karl Moritz Hermann, Gábor Melis, and Edward Grefenstette. 2017. The narrativeqa reading comprehension challenge. Transactions of the Association for Computational Linguistics, 6:317-328.

Chin-Yew Lin. 2004. Rouge: A package for automatic evaluation of summaries. In $A C L$.

Kevin Lin, Oyvind Tafjord, Peter Clark, and Matt Gardner. 2019. Reasoning over paragraph effects in situations. ArXiv, abs/1908.05852.

Preksha Nema and Mitesh M. Khapra. 2018. Towards a better metric for evaluating question generation systems. In EMNLP.

Simon Ostermann, Michael Roth, Ashutosh Modi, Stefan Thater, and Manfred Pinkal. 2018. Semeval2018 task 11: Machine comprehension using commonsense knowledge. In SemEval@NAACL-HLT.

Kishore Papineni, Salim Roukos, Todd Ward, and WeiJing Zhu. 2001. Bleu: a method for automatic evaluation of machine translation. In $A C L$.

Matthew E. Peters, Mark Neumann, Mohit Iyyer, Matt Gardner, Christopher Blake Clark, Kenton Lee, and Luke S. Zettlemoyer. 2018. Deep contextualized word representations. volume abs/1802.05365.

Pranav Rajpurkar, Jian Zhang, Konstantin Lopyrev, and Percy Liang. 2016. Squad: 100, 000+ questions for machine comprehension of text. In EMNLP.

Min Joon Seo, Aniruddha Kembhavi, Ali Farhadi, and Hannaneh Hajishirzi. 2017. Bidirectional attention flow for machine comprehension. In ICLR.

Alon Talmor, Jonathan Herzig, Nicholas Lourie, and Jonathan Berant. 2018. Commonsenseqa: A question answering challenge targeting commonsense knowledge. In NAACL-HLT.

Johannes Welbl, Pontus Stenetorp, and Sebastian Riedel. 2017. Constructing datasets for multi-hop reading comprehension across documents. Transactions of the Association for Computational Linguistics, 6:287-302.

An Yang, Kai Liu, Jing Liu, Yajuan Lyu, and Sujian Li. 2018. Adaptations of rouge and bleu to better evaluate machine reading comprehension task. ArXiv, abs/1806.03578.

Tianyi Zhang, Varsha Kishore, Felix Wu, Kilian Q. Weinberger, and Yoav Artzi. 2019. Bertscore: Evaluating text generation with bert. ArXiv, abs/1904.09675. 